Gerión. Revista de Historia Antigua

ISSN: 0213-0181

\title{
Tiberio y el núcleo romano de Caldes de Montbui (Barcelona). Evidencias de una relación de patrocinio imperial en el conventus Tarraconensis
}

\author{
Joan Oller Guzmán ${ }^{1}$
}

Recibido: 28 de febrero de 2017 / Aceptado: 14 de enero de 2018

Resumen. Este artículo ofrece una interpretación sobre un epígrafe documentado en el núcleo de Caldes de Montbui (IRC I, 40) que podría demostrar la existencia de una relación de patrocinium entre este asentamiento y el emperador Tiberio en época romana. Para dar apoyo a esta hipótesis el artículo analiza las características del enclave termal romano de Caldes, su promoción en época de Augusto y su posible transformación en municipio flavio, convirtiéndose en uno de los puntos centrales en la integración del territorio de la Layetania interior dentro de la estructura provincial romana.

Palabras clave: patrocinium; Tiberio; Layetania interior; Caldes de Montbui; municipium.

\section{[en] Tiberius and the Roman settlement of Caldes de Montbui (Barcelona).} Testimonies of an imperial patrocinium relation in the conventus Tarraconensis

Abstract. This paper offers an interpretation of an inscription documented in Caldes de Montbui (IRC I, 40) which could demonstrate the existence of a relationship of patrocinium between this settlement and the emperor Tiberius. To support this hypothesis we analyze the characteristics of the Roman thermal centre of Caldes, its promotion in Augustan period and its possible conversion in Flavian municipium, being one of the central points in the integration of the territory of Layetania interior in the Roman provincial structure.

Keywords: Patrocinium; Tiberius; Layetania interior; Caldes de Montbui; Municipium.

Sumario. 1. El documento epigráfico. 2. El contexto geográfico e histórico. 3. Tiberio e Hispania. 4. ¿Tiberio, patrono de la civitas termal de Caldes de Montbui? 5. Conclusiones. 6. Referencias bibliográficas.

Cómo citar: Oller Guzmán, J. (2018): Tiberio y el núcleo romano de Caldes de Montbui (Barcelona). Evidencias de una relación de patrocinio imperial en el conventus Tarraconensis, en Gerión 36/1, 185-204.

\footnotetext{
1 Universitat Autònoma de Barcelona/Departamento de Ciencias de la Antigüedad y la Edad Media.

E-mail: joan.oller@uab.cat
} 
El objetivo de este artículo es analizar y contextualizar un epígrafe procedente de la ciudad de Caldes de Montbui (Vallès Oriental, Barcelona, España), el cual ha sido recientemente objeto de una nueva lectura e interpretación. Se trata de una dedicatoria al emperador Tiberio que, según los editores, atestiguaría la existencia de un patrocinio imperial a algún núcleo de esa zona. El interés de esta nueva propuesta radica, por un lado, en la escasez de este tipo de documentos en el territorio español y, por otro lado, en la importancia que este supuesto patrocinio podría tener para interpretar la evolución política y social del núcleo romano de Caldes de Montbui, remarcable por su conjunto termal de época imperial y, por extensión, de la zona donde se sitúa, la Layetania interior, en un momento crucial que supuso su integración definitiva dentro de la organización provincial romana.

\section{El documento epigráfico}

Como decíamos, el epígrafe -correspondiente a IRC I, 40- consiste en una dedicatoria al emperador Tiberio que se ha conservado en la iglesia parroquial. Consiste en un bloque de piedra arenisca reutilizado y recortado por la derecha, quizá correspondiente al friso de un pórtico, situado en un ángulo del edificio en el cual se sitúa la capilla de la Santa Majestat de Caldes de Montbui. La transcripción y traducción del documento es la siguiente: ${ }^{2}$

\section{Ti(berio) $\cdot$ Caesa[ri Divi] \\ [Au]g(usti) $\cdot f($ ilio) $[$ Aug(usto), Augusto] \\ [p]atr[ono]}

“A Tiberio César Augusto, hijo de Augusto divinizado, patrono".

El epígrafe, a nuestro entender, presenta dos problemas básicos: por un lado, por lo que respecta a la lectura e interpretación del texto y, por otro, en relación con su posible documentación in situ. ${ }^{3}$ En cuanto al primer aspecto, vemos que la restitución del término patrono en la última línea no es la única lectura posible $\mathrm{y}$, de hecho, en $I R C$ I, 40 ya se comenta la posibilidad de leer $A T E$ en lugar de $A T R$, secuencia que podría sugerir la restitución del término [potest]ate. A pesar de ello, los editores prefieren decantarse por la primera lectura, puesto que la referencia a la potestad tribunicia del emperador exigiría una nomenclatura y titulación más detallada en el epígrafe. ${ }^{4}$ De hecho, otros autores han compartido la interpretación del epígrafe como un patrocinio imperial como la más probable, si bien planteando alternativas en algunos aspectos. Así, tenemos el caso de Cardon quien de forma general acepta la propuesta de $I R C$ en cuanto al contenido del epígra-

Seguimos la transcripción y traducción realizada por Fabre, Mayer y Rodà en IRC I, 40.

La restitución del epígrafe se llevó a cabo en dos momentos, puesto que al retirar parte del mortero que cubría la pared del edificio en el que se encuentra reutilizado se pudo recuperar la última línea de la inscripción, que hasta ese momento no se conocía. De este modo, en $I R C$ V se llevó a cabo la autopsia completa del epígrafe, hecho que permitió esclarecer en buena parte las dudas y problemas que planteaba, dejando al descubierto el término clave de patronus. Véase IRC $\mathrm{V}$ ad. IRC I, 40.

4 Especialmente teniendo en cuenta la existencia de diversos epígrafes en esta zona layetana interior con referencia a la potestad tribunicia, todos ellos ofreciendo esta estructura con una nomenclatura detallada de todos los cargos y atribuciones del emperador, como por ejemplo en el caso de la dedicatoria a Antonino Pío en Terrassa (IRC I, 66). También Mayer - Rodà 1987, 195. 
fe, pero que discute su estructuración. ${ }^{5}$ Así, propone variantes en relación a la nomenclatura del emperador y a la disposición del epígrafe. En este sentido, considera que el hallazgo del epígrafe en dos bloques separados permite pensar más bien en una estructuración del texto en dos líneas, en lugar de tres, lo cual, para permitir una correcta disposición centrada del mismo, podría haber implicado un mayor desarrollo de la filiación del emperador, hecho bien documentado en los otros ejemplos de patrocinios de Tiberio atestiguados tanto en la Bética como en la Tarraconense. Igualmente, se apoya en el hecho de que lo más probable es que el texto corresponda al friso de un edificio público, lo cual haría que una disposición en dos líneas tuviera mayor lógica. Por tanto, en resumen, la propuesta de restitución de Cardon es la siguiente:

\section{TI. CAESA[R . DIVI . AV]G. F . [DIVI . IVLI . N . AVGVSTVS] [P]ATR[ONVS]}

En el ámbito cronológico, la referencia al divinizado Augusto implica un terminus postquem situado en el 14 d.C. A la vez, la titulatura como Tiberius Caesar y no como Tiberius Iulius Caesar, esta última utilizada por Tiberio entre su adopción el 4 d.C. y la muerte de Augusto, refuerza esta visión. Desgraciadamente no se puede precisar más acerca de la datación del epígrafe dentro del gobierno de Tiberio, si bien Cardon propone la posibilidad de una datación alta dentro de éste. ${ }^{6}$

Por lo que respecta a la cuestión de la localización de la inscripción, la piedra se encontró reaprovechada en un ángulo del edificio parroquial de Caldes, dentro de la capilla de la Santa Majestat. El inusual hecho de documentar un epígrafe de este tipo en un núcleo como el de Caldes ha llevado a algunos autores a plantear la posibilidad de que éste no fuera el emplazamiento original de la inscripción en época romana. ${ }^{7}$ Entre sus argumentos se subraya el hecho de que el material en que está realizado el epígrafe sea arenisca de la montaña de Montjuïc, un dato que sugeriría algún tipo de relación con la ciudad de Barcino. Ahora bien, creemos que éste no es un argumento sólido para descartar una vinculación con el núcleo de Caldes. ${ }^{8}$ Sin embargo, ha sido una idea bastante repetida y que ha condicionado también la interpretación de otras inscripciones fundamentales para entender las características de la civitas termal de Caldes; tal es el caso, por ejemplo, de un epígrafe dedicado a la diosa Isis, ${ }^{9}$ al que posteriormente aludiremos. Por otro lado, los editores de la inscripción alertaban ya sobre el hecho de que vincular este epígrafe con Barcino planteaba un problema básico y es que este emperador nunca recibió honores en esta ciudad layetana. ${ }^{10}$

En nuestra opinión, parece más razonable considerar la pertenencia del epígrafe al núcleo de Caldes por dos motivos principales: en primer lugar, en esta zona se atestigua una amplia tradición de documentos epigráficos romanos, que

\footnotetext{
Para esta propuesta, véase Cardon 2004, 52-53.

Cardon 2004, 52.

De hecho, los propios editores se plantean esta duda (véase IRC V ad. IRC I, 40).

En este sentido, podemos ver cómo se pueden encontrar otros casos de epígrafes hechos en piedra arenisca de Montjuïc en otros asentamientos de la Layetania interior, para los que no se duda de su descubrimiento in situ, como por ejemplo IRC I, 50, en Rubí, o IRC I, 62, en Sabadell. Del mismo modo, buena parte de los epígrafes recuperados en este territorio están realizados en piedra de Santa Tecla, procedente de las canteras tarraconenses, y no por ello se asocian a la ciudad de Tarraco. Sin ir más lejos tenemos los casos de dos documentos epigráficos de Caldes de Montbui (IRC I, 33 y 34), realizados con este material y vinculados claramente al núcleo termal.
}

9 IRC I, 36.

$10 \quad$ IRC I, 40, n. 66. 
empieza ya a finales del siglo II a.C. y que se intensifica durante el siglo I d.C., configurando un magnífico corpus vinculado al centro termal. ${ }^{11}$ En segundo lugar, también justificaría la presencia de esta inscripción in situ la categoría del propio núcleo romano de Caldes, el cual creemos que tuvo estatus de civitas ya desde época augustea, posiblemente transformándose en el ámbito jurídico en municipio en época flavia. Esta cuestión será analizada con mayor detalle más adelante.

Aunque no pretendemos ignorar estas problemáticas, creemos plausible partir de la hipótesis de trabajo según la cual estamos ante una dedicatoria al emperador julio-claudio Tiberio en la que se atestigua una relación de patrocinio con algún núcleo local. ${ }^{12} \mathrm{El}$ hecho de que se trate de un friso de pórtico conduce a pensar, a la vez, que podría haber formado parte de un edificio monumental, quizás erigido por un miembro de la élite local en relación con este patronato imperial. Por otro lado, si tenemos en cuenta la existencia, dentro del corpus epigráfico de Caldes, de otra inscripción, datada en el cambio de era, consistente en una dedicatoria a la diosa Isis y que hace referencia a una res publica, ${ }^{13}$ entonces podríamos afirmar sin duda que la época augusteo-tiberiana fue clave para el desarrollo de este núcleo. En relación con el epígrafe dedicado a Isis existe una problemática inherente relativa a si realmente es una inscripción originaria de Caldes o si bien fue transportada y reaprovechada desde otro núcleo como Tarraco. En nuestro caso, creemos plausible que fuese originario de Caldes en base a la posibilidad, que más adelante comentaremos, de que el núcleo romano de Caldes fuese creado en época augustea y promovido desde ese momento a una categoría jurídica romana. ${ }^{14}$ Por otro lado, cabe remarcar la contrastada y frecuente relación de la diosa Isis con los centros termales, bien atestiguada en casos como el municipium de Aquae Flaviae (Chaves, Portugal), el núcleo de Aquae Helvetiorum (Baden, Suiza), Aquae Calidae (Vichy) y Neris-les-Bains en Francia, Aquae Aureliae (BadenBaden, Alemania) o Aquae Flavianae (Henchir Hammam, Argelia). Así, Isis tendría entre sus diversas atribuciones la de divinidad de la salud, de tal modo que, por ejemplo, acostumbra a aparecer asociada con otras divinidades curativas como Salus o Hygieia. En este sentido, pues, pensamos que resulta perfectamente lógica la presencia de una inscripción vinculada a Isis en el contexto del centro termal de Caldes, acompañada de otras referencias a Salus, Minerva o Apolo, divinidades también habitualmente encontradas en estos contextos salutíferos. Por tanto, la evidente relación de las aguas termales de la zona de Caldes con procesos curativos vinculados a personajes de cierta relevancia social procedentes de Barcino o Tarraco, atestiguada en la epigrafía recuperada en el conjunto termal, permite dar fundamento a la hipótesis de que el epígrafe dedicado a Isis podía haber

11 Disponemos de un miliario del procónsul Manius Sergius datado hacia el 120 a.C. (IRC I, 181) recuperado en el cercano municipio de Santa Eulàlia de Ronçana y asociado a la importante reforma viaria que sufrió esta zona a finales del siglo II a.C. Por otro lado, en vinculación con el centro termal y su zona circundante, se han recuperado un total de once documentos epigráficos, constituyendo uno de los conjuntos más remarcables del Vallès, sólo por detrás de los vinculados al municipio romano de Egara (Terrassa, Vallès Occidental). Véase: Mayer - Rodà 1984, 51 y 53.

12 Hipótesis también postulada por otros autores como Cardon 2004, ya mencionada, o Melchor Gil 2014, 263 , n. 35 .

13 Véase supra, n. 9.

14 Una hipótesis reafirmada por sus editores en los trabajos más recientes de restitución del documento (IRC V ad. IRC I, 40). 
estado originariamente situado en dichas termas. ${ }^{15}$ Cabe remarcar que existe otra posible dedicatoria imperial recuperada en Caldes, en este caso dedicada a Septimio Severo, un pedestal procedente de la zona termal del núcleo romano de la ciudad, desgraciadamente perdido. ${ }^{16}$

\section{El contexto geográfico e histórico}

El núcleo romano de Caldes de Montbui ${ }^{17}$ se situaría dentro del territorio denominado Layetania interior, una zona que abarcaría la parte interna de la "región ibérica" habitada por el pueblo layetano, bien conocido e identificado gracias a las fuentes literarias clásicas ${ }^{18}$ y que correspondería, grosso modo, a las actuales comarcas del Vallès Occidental y Oriental (al norte de Barcelona). Desde antiguo, la importancia de este territorio ha venido determinada por dos características básicas: su papel como zona viaria clave y su elevada productividad agrícola. ${ }^{19}$ Estas características hicieron que, desde un primer momento, Roma estuviera altamente interesada en su control y, de hecho, las fuentes muestran que fue una de las primeras zonas en ser sometidas por las legiones romanas en la Península Ibérica. ${ }^{20} \mathrm{~A}$ partir de este momento, la Layetania interior fue evolucionando del mismo modo que el resto de territorios hispanos conquistados en el noreste peninsular, adaptándose progresivamente a las estructuras sociales, económicas y políticas del Imperio romano. Con todo, creemos que, del mismo modo que para el resto de Hispania, el momento clave de este proceso fue el período augusteo. En este sentido, la arqueología demuestra claramente que éste es un momento de transformación y reordenación en la zona layetana, ${ }^{21}$ en concordancia con los cambios generales acontecidos en las provincias hispanas. ${ }^{22}$

Así, durante este período se produce la integración definitiva de la Layetania interior en las estructuras imperiales romanas, acaeciendo todo un conjunto de transformaciones que marcan los ejes de la actuación para el control y la explotación de este territorio por parte de Roma. Principalmente, podemos resumirlas en la reforma viaria, la centuriación del territorio, la implantación de un modelo económico basado en la villa y la creación de núcleos urbanos vertebradores del territorio. ${ }^{23}$ Para nues-

15 Visión que comparten diversos autores, como Malaise 1984, 1650, y Díez de Velasco 1996, 146-148. Con todo, defendiendo un origen en Tarraco, Alvar 1993, 29, n. 14. Cf. Mayer i Olivé 2010, 306 y n. 22.

16 Véase IRC I, 41 .

17 Aunque tradicionalmente se ha denominado Aquae Calidae, no existe ninguna evidencia ni en las fuentes clásicas ni en las epigráficas que lo certifique, de tal modo que desconocemos cuál fue el nombre real en época romana de este asentamiento.

18 Partiendo básicamente de las referencias de Estrabón (3.4.8), Plinio el Viejo (HN 3.3.21), Ptolomeo (2.6.18, 6.72) y, en menor medida, de Pomponio Mela (2.90) y Avieno (Ora 519-522).

19 No en vano sabemos que a través de ella pasaba la vía Augusta y, por ejemplo, las intervenciones realizadas en el núcleo de Sabadell (Vallès Occidental) han permitido identificar parte del recorrido de esta vía (Roig i Buxó 2002, 76-91, y 2004, 809-823; Flórez - Rodà 2014; Oller Guzmán 2015, 231-246). Mientras que, por otro lado, tanto la arqueología como las fuentes clásicas indican la existencia de una importante producción vitivinícola a partir del siglo I a.C. en esta zona con dos producciones diferenciadas: el vino layetano y el vino de Lauro, cuestión que trataremos posteriormente con más detalle.

20 Ya desde las campañas de Cneo Cornelio Escipión el 218 a.C. (Plb. 2.76.1-2; Liv. 21.60.1-4).

21 Cuestión que ya hemos tratado in extenso en otros trabajos (Oller Guzmán 2009, 193 y ss., y 2016, 137-175).

22 Reflejados tanto en la reforma provincial como en la fundación de ciudades (Augusta Emerita, Caesar Augusta, Barcino, etc.) o en la reordenación del territorio (con el caso paradigmático de Augusta Emerita). Véase Salinas de Frías 1995, 93-98; Barroso et alii 2003, 93-104; Gorges - Rodríguez Martín 2005, 93-128.

23 Para una visión detallada de estos ejes: Oller Guzmán 2009, 202 y ss., y 2016. 
tro caso de estudio, resulta especialmente interesante el último elemento enumerado, la creación de núcleos urbanos, puesto que está en relación directa con el núcleo de Caldes. Así, sabemos que Augusto fundó en la costa layetana la colonia de ciudadanos romanos de Barcino, para ejercer de punto central de esta área del noreste de la Península Ibérica. En la zona interior, con todo, la arqueología no ha podido comprobar la existencia de ninguna fundación urbana clásica en época augustea. A pesar de ello, la importancia estratégica de esta zona, tanto viaria como económicamente, hacía que fuera difícil que quedase desprovista de núcleos centrales de control y organización. De este modo, Roma buscó formas alternativas de control que no implicasen la necesaria fundación de ciudades urbanísticamente definidas y lo consiguió mediante la promoción de pequeños núcleos indígenas preexistentes a los que otorgó la función de centros vertebradores del territorio en base a la reordenación ejercida por Augusto. Estos centros no tendrían la necesidad de desarrollar un entramado urbano para ejercer dicha función, puesto que se servían de un pequeño núcleo monumentalizado con uno o varios edificios para realizar las actividades políticas, administrativas, de mercado o incluso religiosas que fueran requeridas, pero con una categoría jurídica romana propia, es decir, como civitas. $^{24}$

En este contexto, en la Layetania interior se promovieron dos núcleos: en la zona occidental, Egara ${ }^{25}$ y, en la central-oriental, el núcleo romano de Caldes de Montbui. En lo que concierne al último caso, el que nos ocupa, a finales del siglo I a.C. se detecta el abandono del principal oppidum ibérico de la zona, el de la Torre Roja, ${ }^{26}$ que pasa a ser sustituido como centro vertebrador del territorio por el nuevo asentamiento de Caldes, donde se construyó un conjunto termal romano del cual aún se conservan remarcables vestigios. ${ }^{27}$ No hay duda, por tanto, de que se trata de un núcleo de promoción augustea, pero resulta muy difícil establecer sus características con claridad por distintas razones: por un lado, la arqueología demuestra por ahora la inexistencia de una ciudad con entramado urbano clásico; por otro, las fuentes literarias no hacen referencia a este asentamiento, mientras que la relativamente abundante epigrafía recuperada en ningún caso menciona directamente el núcleo, de tal modo que no podemos saber ni siquiera su nombre exacto.

A pesar de ello, existen algunos elementos que subrayan su importancia, más allá del conjunto termal en sí mismo. Por un lado, el surgimiento de una abundante red

24 Es esta una tipología que se puede encontrar de forma abundante por todo el Imperio y que hemos venido a denominar como "civitas sine urbe". Se trata de un concepto historiográfico moderno y que, por ejemplo, también se ha utilizado como "civitas sine oppido", la "no ciudad", "agglomérations secondaires" en la historiografía francesa o "small towns" en la anglosajona. Si bien no aparece como tal directamente en las fuentes clásicas, éstas sí que hablan de forma bastante habitual de estas realidades "no urbanas". Sobre el concepto véase Arrayás et alii 2001, 311-317; Cerrillo Martín de Cáceres 2003, 49; Prieto Arciniega 2008, 28-30; Oller Guzmán 2014 , 89-110.

25 El caso de Egara resulta particularmente interesante, con la identificación del núcleo en un punto estratégico donde se han documentado diversos restos arqueológicos, junto a una acumulación de epígrafes honoríficos que confirman la existencia de la civitas, la cual obtuvo en época flavia la categoría municipal. Para el caso de Egara véase Prieto Arciniega 1997, 209-215; Olesti Vila - Garcia Llinares 1999, 13-23; Garcia i Llinares et alii 2009, 33-34; Oller Guzmán 2009, 189-208; 2014, 98 y ss., y 2015, 290 y ss.

26 El poblado ibérico de la Torre Roja, situado en una elevación estratégica y con una ocupación desde el Ibérico Antiguo, es uno de los principales oppida de toda la Layetania interior, con un papel clave de control sobre los accesos desde la zona layetana hacia la parte central del noreste peninsular. Acerca este asentamiento, entre otros, véase Fortó et alii 2005, 5-18; Fortó Garcia - Maese Fidalgo 2012, 113-152.

27 No entraremos en detalle sobre estos vestigios, remitiendo a los principales trabajos descriptivos existentes al respecto, tales como Miró i Alaix 1992a, 255-276, y 1992b, 11-29. 
de villae y de asentamientos productivos rurales alrededor de Caldes, a partir precisamente del cambio de era, red vinculada a la producción vitivinícola y con una denominación de origen documentada tanto en las fuentes clásicas como mediante la epigrafía anfórica. ${ }^{28}$ Por otro lado, el corpus epigráfico, que permite plantear la hipótesis de la existencia de un núcleo romano jurídicamente establecido desde época augustea, promocionado a municipio romano en época flavia. ${ }^{29}$

Por tanto, estamos ante un enclave que, por sus características, se puede convertir en un magnífico ejemplo de la actuación romana de ordenación, estructuración y control de los territorios provinciales hispanos mediante la adaptación y transformación de las realidades indígenas preexistentes; es decir, del complejo y poliédrico "proceso de romanización". Pero, a la vez, la falta de certezas absolutas alrededor de buena parte de los datos de que disponemos sobre el núcleo de Caldes, hacen que el citado epígrafe con el patrocinio imperial resulte, creemos, de fundamental importancia no sólo para entender la propia evolución de la civitas termal, sino también la romanización del noreste de la Península Ibérica.

\section{Tiberio e Hispania}

El otro punto para el cual, obviamente, este epígrafe resulta interesante sería para documentar la actividad del emperador Tiberio en la Península Ibérica y, más concretamente, en su cuadrante noreste. Es por ello que primero debemos considerar breve-

28 En concreto, Plinio habla del vino Lauronensis (HN 14.71), diferenciándolo del vino layetano por su mayor calidad. De hecho, otros autores, como Marcial, ya indicaron la importancia de esta actividad productiva en la zona layetana, remarcando una apuesta por la alta producción, más que por la finura de ésta (Mart. Ep. 1.26.910; 7.53.1-10). Para poder situar esta Lauro vinícola en la zona cercana a Caldes de Montbui existen diferentes evidencias. La más importante sería la que aporta la epigrafía anfórica. Así, se han documentado diferentes ánforas vinícolas Dressel 2/4 (de origen tarraconense) con la presencia de tituli picti con las palabras Lavr-, Lavr/ ve (Lauro vetus) y Lavr/IIII en zonas como Roma, Ostia o Cartago. Por tanto, estaríamos ante una producción vitivinícola de origen tarraconense, con denominación de origen lauronensis y de notable calidad, tal como muestran los distintivos vetus o IIII, indicativos de ser un vino de cierta antigüedad. A ello le tenemos que añadir el hecho de que el análisis de la pasta cerámica de las ánforas mostró una gran similitud con la documentada en los alfares situados alrededor de Caldes. Si a todo ello le sumamos la evidencia arqueológica que certifica el papel destacado de esta zona como productora vitivinícola y alfarera, parece probable que la producción de vino de Lauro se situaría en diversos establecimientos de la zona de Caldes y su área circundante. Finalmente, se debe añadir a todo ello la contrastada existencia de la ceca monetaria ibérica de Lauro en algún punto de esta zona del Vallès Oriental. Dicha ceca se vincularía a un núcleo ibérico de primer orden con una fase republicana también remarcable. Hasta este momento no se había podido identificar dicho asentamiento, pero en los últimos años las excavaciones arqueológicas en el yacimiento de Puig del Castell, en la cercana localidad de Cànoves i Samalús, podrían haber permitido encontrar la Lauro ibérica, ofreciendo un indicio definitivo para situar en este contexto territorial la futura Lauro vinícola. Para más detalles acerca de toda esta cuestión, véase Pascual 1998, 149-162; Aguilar 2005, 5-12; Oller Guzmán 2015, 434-474. Sobre la ceca monetaria, véase Estrada - Villaronga 1967, 134-194; Llorens Forcada - Ripollès Alegre 1998. En relación con la Lauro ibérica cf. Guàrdia i Llorens 2014, 75-91, y 2015, 51-86.

29 Dos son los epígrafes que permiten plantear esta hipótesis. Por un lado, tenemos la citada dedicatoria a la diosa Isis (IRC I, 36) datada en el cambio de era y recuperada cerca del conjunto termal, donde se hace referencia a una res publica que podría ser el mayor indicio en referencia a la promoción jurídica del núcleo de Caldes en época augustea. Por otro lado, un epígrafe dedicado a un tal Lucius Caecilius Serenus o Seranus por parte de su hijo o liberto (IRC I, 42), recuperado en una capilla de Caldes y en donde se especifica su cursus honorum con cargos como edil, duunviro y flamen. Con una cronología entre la época flavia y la segunda mitad del siglo I d.C., constituiría la mayor evidencia disponible para poder hablar de un municipio de promoción flavia en esta zona, juntamente al caso cercano y prácticamente paralelo del ya citado núcleo de Egara. Para un anàlisis de estos epígrafes, sus problemáticas e interpretación, véase Oller Guzmán 2015, 285 y ss. 
mente el papel de este emperador en las provincias hispanas. Así, durante su período de gobierno (14-37 d.C.) de forma general se le atribuye la continuación y consolidación de las acciones de Augusto, especialmente importantes en las zonas de más reciente conquista como las del noroeste peninsular, anexionadas en el año 19 a.C. y finalmente incluidas en la Hispania Citerior. ${ }^{30}$ En este sentido, por ejemplo, cabe remarcar la continuidad de la actividad viaria, que contribuiría a mantener el impulso iniciado por Augusto en esta dirección en toda la Península. Prueba de ello serían los 37 miliarios de Tiberio recuperados en Hispania, documentados en las tres provincias, pero con un claro predominio de la Tarraconense, donde se acumulan 31 miliarios del total. ${ }^{31}$ Este hecho afecta directamente a la Layetania interior, donde se ha recuperado precisamente uno de estos miliarios de Tiberio. ${ }^{32}$

De hecho, parecería que las actuaciones tiberianas tuvieron especial repercusión en esta provincia Tarraconense, provincia sobre la que tenía un mayor conocimiento después de haber desarrollado sus primeros cargos militares en ella. ${ }^{33}$ Posiblemente ello hizo que impulsara programas edilicios en sus ciudades, especialmente en aquellas recientemente promovidas por Augusto, contribuyendo a su consolidación y a la del sistema socio-económico y administrativo que representaban. En el noreste peninsular, quizá el testimonio más interesante lo aporten las fuentes literarias, en concreto Tácito, cuando indica cómo, en el año 15 d.C., el emperador autorizó la construcción de un templo de culto imperial a Augusto en Tarraco. ${ }^{34}$ Tradicionalmente situado bajo la actual catedral de la moderna ciudad de Tarragona, a día de hoy todo parece indicar que realmente se creó un centro cultual de primer orden en este punto de la antigua Tarraco, con unos inicios enmarcados, como mínimo, dentro de la primera mitad del siglo I d.C. ${ }^{35}$

Esta actividad edilicia no se limitó sólo a la capital provincial, sino que se documenta también en otros núcleos claves como por ejemplo Caesar Augusta, entre otros. ${ }^{36}$ Igualmente, en el noreste peninsular podríamos citar el caso de Dertosa, donde la documentación de unas acuñaciones de época de Tiberio habría hecho plantear que, en ese momento, el núcleo fuera promovido a categoría colonial, razonamiento a día de hoy rechazado. ${ }^{37}$ De hecho, de forma general los autores que han tratado sobre este período subrayan una posición política más bien conservadora de

30 Arrayás 2006, 191.

31 De hecho, es el emperador de esta dinastía que, de largo, ha aportado más hitos viarios. Véase Lostal Pros 1992; Solana Sáinz - Sagredo San Eustaquio 2006, 35-36, y 2008, 52-58.

32 Miliario registrado en $I R C \mathrm{~V}, 235$.

33 Concretamente como tribuno militar durante la campaña de las Guerras Cántabras (Suet. Tib. 9).

34 Tac. Ann. 1.78.

35 Precisamente en estos últimos años se ha estado desarrollando un plan de intervenciones arqueológicas en la catedral de Tarragona con unos resultados ciertamente interesantes. En relación con ello y sin ánimo de exhaustividad, véase Macias et alii 2007a, 765-787; Macias et alii 2007b, 151-213; Casas et alii 2008, 9-12; Macias Solé et alii 2012a y 2012 b.

36 Para el caso de Caesar Augusta: Guerrero Arroyo 1993, 264; Casabona Sebastián - Pérez-Casas 1994, 91-92; Escudero - Galve 2006, 189-197. Habría más ejemplos de esta actuación en época tiberiana. Así, en la Tarraconense encontramos el caso de Bilbilis: Martín-Bueno - Sáenz Preciado 2004, 257-276. En la Lusitania podemos hablar del caso paradigmático de Augusta Emerita: Trillmich 2004, 321-335; Alba Calzado - Mateos Cruz 2006, 153-159. Finalmente, en la Bética podemos citar el caso de Iponuba: Castillo - Ruiz-Nicoli 2008, 149-186.

37 Puesto que se han rechazado las citadas evidencias numismáticas. Se considera, pues, que estaríamos ante un municipio promovido bajo César o Augusto. Véase Guerrero Arroyo 1993, 97; Genera i Monells - Vianney Arbeloa 1987, 81-90; Genera i Monells 1994, 171; Mayer i Olivé 2009, 61-69. 
Tiberio por lo que respecta a las promociones jurídicas de comunidades peregrinas hispanas, frenando de algún modo la municipalización de la etapa anterior. ${ }^{38}$ Con todo, otros autores consideran que bajo Tiberio se daría la última fase de este proceso de municipalización, con el surgimiento de municipios como Leonica, Cascantum, Grachurris, Uxama, Termes o Clunia, siendo a partir de Calígula cuando se puso freno de forma general de este fenómeno. ${ }^{39}$

Sea como sea, y con mayor o menor intensidad, parece que habría un acuerdo general en el hecho de que, bajo Tiberio, se dio una continuidad del proceso de reformas iniciado por Augusto y, por tanto, en el intento de integrar de forma definitiva Hispania dentro del funcionamiento del Imperio. Desgraciadamente, las fuentes literarias son excesivamente parcas a partir de época imperial a la hora de referirse al territorio hispano, existiendo sólo referencias escasas relacionadas con la actividad de los propios emperadores, pero no con la organización y funcionamiento de las provincias hispanas. Es por ello que resulta complejo poder seguir con exactitud las actuaciones tiberianas en dichas provincias y el interés que por ellas pudo mantener; si bien tanto la arqueología, como la epigrafía y, en menor medida, las fuentes literarias, dan fe de que esta intervención existió. De este modo, por ejemplo, son relativamente abundantes los epígrafes honoríficos disponibles en la Península Ibérica dedicados a Tiberio, pero pocos de ellos permiten inferir una actuación directa del poder imperial. ${ }^{40}$ Por ello resulta tan importante un documento epigráfico como el que aquí se analiza, puesto que un patrocinio imperial suponía un vínculo directo de un núcleo con la persona del propio emperador.

\section{4. ¿Tiberio, patrono de la civitas termal de Caldes de Montbui?}

Por tanto, tenemos un epígrafe que pensamos que se puede relacionar con el centro termal romano de Caldes de Montbui y que, a la vez, vincula a éste con el emperador Tiberio. A partir de aquí, queda intentar relacionar ambos elementos para poder entender por qué un emperador de la dinastía julio-claudia se podría haber interesado por este pequeño núcleo de la Layetania interior.

En primer lugar, cabe situar la cuestión de los patrocinios imperiales en su debido contexto, puesto que los patrocinia se documentan por todo el Imperio. ${ }^{41}$ Habitualmente este tipo de inscripciones consisten en un breve y conciso epígrafe donde sólo se menciona el nombre, la carrera y los títulos del patrono, adjuntando a veces una

38 Comparten esta visión autores como Henderson 1942, 2; Alföldy 1965, 824-844, o Santos Yanguas 1989, 112-113.

39 Abascal - Espinosa 1989, 68.

40 Para un repaso sobre los diferentes epígrafes honoríficos dedicados a Tiberio, véase: Rodríguez Oliva - Beltrán Fortes 1986, 223-224. Un caso bastante reciente e interesante sería el de la ciudad de Termes, donde -en las intervenciones del año 2004- se documentó un epígrafe honorífico dedicado a Tiberio en la zona del foro, mostrando también la importancia de este preciso momento en el crecimiento edilicio de la ciudad (véase Mangas - Martínez Caballero 2004, 289-300).

41 La cuestión del patronazgo en el mundo romano ha sido ampliamente debatida por parte de la historiografía y no entraremos en detalle en lo relativo a las problemáticas generales que supone. Para un repaso sobre las principales obras que han tratado esta cuestión, remitimos a las referencias aportadas en los trabajos de Lintott 1993, 174, o Eilers 2002, 1-18, y a la obra básica en relación con el patronazgo cívico de Nicols 2014, 5-7. 
fórmula honorífica que rendiría homenaje al personaje en cuestión. ${ }^{42}$ L. Harmand, en su ya clásico trabajo sobre los patrocinios en época romana, remarca que estos patrocinios imperiales debían de ser los más deseados por parte de los diferentes núcleos del Imperio, si bien tenemos muy pocos ejemplos epigráficos; mucho más frecuente, sin embargo, es el patrocinio de magistrados vinculados tanto a la administración imperial como, especialmente, a la provincial. ${ }^{43}$ En general, dichos patrocinios implicaban una relación en la que la comunidad que los recibía buscaba obtener un intermediario que permitiese defender sus intereses en Roma, consiguiendo algún beneficio que raramente se especificaba en el texto epigráfico. ${ }^{44}$ En el caso de los patrocinios imperiales, evidentemente implicaban un vínculo directo con la dinastía gobernante en Roma y, por tanto, suponían un gran prestigio y, en principio, una mayor benevolencia por parte del poder central. A la vez, dichas relaciones tenían una carga religiosa importante, puesto que aceptar un patrocinio del emperador o de miembros de la casa augusta implicaba una aceptación tácita del modelo político del Principado, el cual era querido y protegido por los dioses. ${ }^{45}$ Normalmente el establecimiento del patrocinio consistía en un proceso voluntario, hecho público por cooptación oficial en forma de decretum decurionum y con la participación, en algún caso, de un intermediario. ${ }^{46}$

Tradicionalmente, se habían asociado estos patrocinios imperiales con la fundación de un nuevo núcleo o con la concesión de una nueva categoría jurídica. Así, ya autores como J. Houdoy defendían que, de hecho, cuando un emperador fundaba una nueva ciudad o le concedía un nuevo estatuto jurídico, pasaba a ser patrón de ésta de forma automática ${ }^{47}$ Con todo, la Lex Coloniae Genetivae Iuliae explica cómo, en principio, se reserva el patrocinio a aquel personaje que tiene el derecho de dar o asignar tierras a los colonos o a aquel que haya deducido la colonia, a sus hijos o descendientes. ${ }^{48}$ A partir de aquí, Harmand plantea que, en realidad, quien llevaba a cabo la deductio efectiva de la colonia era un magistrado (normalmente un legatus imperial) y, por tanto, en todo caso el patrocinio tenía que ser para este magistrado y no para el emperador. ${ }^{49}$ En realidad, podemos considerar que el patrocinio y las clientelas, a partir del período tardorepublicano, se convirtieron en un instrumento de dominación política, utilizado por personajes como Pompeyo, Marco Antonio o César. Este último, de hecho, fue declarado patrón de toda la ciudad de Roma y del Estado entero. ${ }^{50}$ Por su parte, Augusto mantuvo esta política, probablemente ante cierto temor a la utilización tendenciosa de esta institución como forma de obtener prestigio y apoyos políticos por parte de aspirantes al poder en Roma. ${ }^{51}$ Esta situación explicaría por qué se puede detectar a partir de este momento una tendencia a dificultar la concesión de patrocinios a personajes situados fuera de la casa imperial,

42 Duthoy remarca cómo, en el caso italiano, se documentan para el Alto Imperio un conjunto de inscripciones de este tipo, de las cuales la gran mayoría (70\%) serían de tipo honorífico, muy por encima de las funerarias y votivas (Duthoy 1984-1986, 122).

43 Harmand 1957, 155.

44 Nicols 1978, 429.

45 Cardon 2004, 39-40.

46 Nicols 1978, 429-430.

47 Houdoy 1876, 254.

48 Rúbrica XCVII.

49 Harmand 1957, 156.

50 DC 44.48.1-2.

51 Melchor Gil 2014, 252. 
básicamente a miembros de la clase senatorial, tal como se puede apreciar en el capítulo CXXX de la Lex Coloniae Genetivae Iuliae. ${ }^{52}$

Así, el Princeps se consolidó como patrón único de todo el Imperio, reforzando su posición como único ostentador del poder en Roma. ${ }^{53} \mathrm{~A}$ la vez, este hecho probablemente implicaría la herencia de las relaciones de patrocinio previamente establecidas por su padre adoptivo, César, ya fuese mediante su reclamación por parte del emperador, o bien a través de una renovación impulsada por las propias comunidades al considerarse a Octaviano como heredero legal y político de éste. ${ }^{54}$ Ello implicaría, pues, que Octaviano se convertiría automáticamente en patrono de los municipios y colonias promocionados por César y, obviamente, este hecho también se haría extensivo a aquellos municipios y colonias promocionados por él mismo. Por tanto, Octaviano utilizaría la institución del patrocinio cívico como forma de afirmar y consolidar su poder, heredando un tipo de actuación habitual en la República tardía, pero a la vez intentando circunscribirla al ámbito de la familia imperial, para evitar posibles usos contrarios a sus intereses.

Esta situación llegó a su punto final a partir del año 2 a.C., cuando Augusto recibe el título de pater patriae. Así, según la hipótesis planteada en su momento por F. Engesser, la concesión de dicho título implicó la promoción de Augusto como protector oficial de todos los habitantes del Imperio, de tal modo que las relaciones de patrocinio entre el emperador y las diferentes comunidades del mismo dejaban de tener sentido. ${ }^{55} \mathrm{~A}$ partir de este momento, pues, no deberíamos encontrar más emperadores de la dinastía julio-claudia que aparecieran como patronos de comunidades del Imperio Romano, si bien aún se podía dar esta relación con otros miembros de la familia imperial, los cuales al no ostentar el cargo de princeps, no podían optar al título de padre de la patria. ${ }^{56}$ Pero entonces, ¿cómo puede ser que encontremos dicho epígrafe asociando un patrocinio de Tiberio con el núcleo romano de Caldes de Montbui? La explicación a esta cuestión sería sencilla, puesto que Tiberio fue el único emperador de la dinastía julio-claudia que no adoptó de forma oficial dicha titulación de pater patriae, de tal modo que en su caso sería lógico seguir documentando este tipo de relaciones de patrocinio con municipios y colonias provinciales, hecho que, como veremos, está bien atestiguado en territorio hispano. ${ }^{57}$

En relación con todo ello, un aspecto importante sería la cuestión de la herencia de estas relaciones de patrocinio. De este modo, y por lo que se refiere a Caldes, cabe preguntarse si este posible patrocinio vinculado a Tiberio fue originalmente creado bajo el gobierno del segundo emperador de la dinastía, o si más bien fue heredado de Augusto ${ }^{58}$ De hecho, uno de los principales problemas a la hora de identificar los epígrafes que hacen referencia a patrocinia imperiales y su cronología, proviene de la posibilidad de que fuese un patrocinio heredado de un emperador anterior. De este

52 Sobre la cuestión de esta lex remitimos a Caballos Rufino 2004 y 2007.

53 Harmand 1957, 158-159. Una visión contraria sería la de Eilers, quien considera que el hecho de que este patrocinio sobre todo el Imperio no esté atestiguado en las fuentes clásicas es un elemento a remarcar. A la vez, defiende que al haber unas cuantas ciudades que reconocen a Augusto como patrón, ello implicaría que, precisamente, no se le consideraba como tal para todo el Imperio (Eilers 2002, 186).

54 Melchor Gil 2014, 253.

55 Engesser 1957, 12-24.

56 Y esta situación se mantendría durante la dinastía flavia y, con algunas excepciones, en época antonina (Cardon 2004, 54).

57 Melchor Gil 2014, 263, n. 35.

58 Sobre la cuestión de la herencia de las relaciones de patrocinio, de forma general, Eilers 2002, 61-83. 
modo, tanto podía ser que un núcleo adoptase como patrón a un emperador antes que éste lo fuera oficialmente, como que lo hiciese cuando ya había adoptado el cargo. ${ }^{59}$ En el caso de Caldes resulta complejo poder determinarlo con certeza, existiendo indicios a favor de ambas hipótesis.

Volviendo al tema que nos ocupa, podemos ver cómo la figura de Tiberio fue patronus de otros núcleos de la Península Ibérica. Así, en la Bética encontramos el caso de Ulia e Italica ${ }^{60}$ mientras que en la Tarraconense destaca el de Carthago No$v a{ }^{61}$ Igualmente, y aunque no aparece como patrón, son remarcables las menciones epigráficas a Tiberio documentadas en Tarraco y Emerita. Así pues, se trataría de unas referencias escasas, que resaltarían dos aspectos. Por un lado, de forma más general, el hecho ya comentado de que estos patrocinios imperiales, tras Augusto, se hicieron cada vez menos habituales, siendo el título de pater patriae el que acabó substituyéndolos o incorporando sus prerrogativas. En segundo lugar, remarca la importancia del citado epígrafe de Caldes, que sería uno de los pocos documentos epigráficos de toda Hispania que podría hacer referencia a un patrocinio de este emperador.

Pero ¿qué podría significar este patrocinio documentado epigráficamente en la Layetania? Parece bastante evidente que no implicaría el paso de Tiberio por esta zona, pues ya hemos visto que, en realidad, Tiberio tuvo una escasa relación directa con Hispania, focalizándose el grueso de su acción en el marco de las campañas contra cántabros y astures. Una de las opciones sería pensar en un traslado y reaprovechamiento del epígrafe desde un núcleo de primer orden cercano como sería Barcino. En este sentido, no haría falta que fuese el propio Tiberio quien hubiese realizado alguna actuación vinculada con la nueva colonia, sino que podría ser sencillamente que hubiese heredado esta condición de patrón de su fundador, Augusto. Ya hemos visto que en las $I R C$ se plantea esta posibilidad, pero también se subraya la falta de honores dedicados a Tiberio en Barcino, dificultando así esta atribución. En cambio, si consideramos que el epígrafe se encontraba originalmente en el asentamiento de Caldes, entonces estaríamos ante un claro indicio de la importancia del conjunto termal desde época augustea. Ya hemos comentado con anterioridad que no existe ningún elemento que aporte una evidencia sustancial que permita pensar en una ubicación original en Barcino. En cambio, el remarcable corpus epigráfico documentado en este núcleo, junto a su hallazgo en la citada capilla de la Santa Majestat, serían buenos indicios para defender su origen en la zona. Así, esta capilla se encuentra a escasos 200 metros del edificio termal, punto de hallazgo de la mayor parte de epígrafes recuperados en Caldes. Por tanto, no resulta ilógico pensar en un reaprovechamiento desde su zona de origen. Es más, tenemos el paralelo cercano del núcleo de Egara, cuyos principales epígrafes (los que certifican la existencia del municipio flavio) se hallan incrustados en la iglesia de Santa María de Égara, mostrando pues un ejemplo idéntico de reaprovechamiento de elementos procedentes de los centros de poder romanos en las posteriores estructuras cristianas situadas en los alrededores.

Asumiendo, pues, esta posibilidad, la problemática sería determinar si este patrocinio provendría de una herencia augustea, teniendo en cuenta nuestra hipótesis so-

59 Sin embargo, por poner un ejemplo con el citado trabajo de Harmand, la mayor parte de los casos analizados por éste corresponden a patrocinia establecidos cuando los patronos eran herederos o candidatos al trono imperial (Harmand 1957, 179-180).

60 Véanse $C I L \mathrm{II}^{2} / 5$, 490, y CIL II 1113 , respectivamente.

61 CIL II 5930. 
bre la potenciación de este centro durante las reformas de Augusto, o si se trataría de una relación que empezaría realmente durante el gobierno de Tiberio. En general, la titulación del epígrafe, que hace referencia a Tiberio como César y a un Augusto divinizado, no deja dudas acerca de su realización, posterior al 14 d.C. Ahora bien, ello no permite discernir sobre si fue una nueva concesión o más bien la renovación del patrocinio en la figura del nuevo emperador, después de la muerte del primer Princeps.

Existen indicios que podrían hablar en favor de ambas opciones. Así, si ya hemos visto la importancia del período augusteo para la Layetania y, más concretamente, para el conjunto termal de Caldes, las actuaciones en época tiberiana en la zona también están documentadas gracias a la recuperación de un miliario del emperador en la localidad de Sant Cugat, en el Vallès Occidental. ${ }^{62}$ En general, es una cuestión que se imbrica de forma irremediable con la evolución del núcleo termal y con su definición jurídica. De este modo, ya hemos planteado el surgimiento del conjunto termal romano de Caldes en el cambio de era y en relación directa con las reformas impulsadas por Augusto en la zona, de forma paralela a lo que sucedía en Egara, en la parte occidental de la Layetania interior, convirtiéndose ambos núcleos en los ejes vertebradores indiscutibles de la actividad política, económica y social del territorio vallesano. Para ello, sería lógico pensar en la constitución, tanto en Caldes como en Egara, de una civitas y, por ende, en su entrada dentro del ámbito jurídico-político romano. En el caso de Caldes, la epigrafía nos brinda un indicio acerca de esta posible promoción gracias al ya citado, aunque polémico, epígrafe de Isis que hace mención a una res publica. ${ }^{63}$ Con todo, el momento jurídicamente definitivo para estos centros sería el período flavio, cuando podrían acceder a la categoría municipal, un hecho bien documentado para el municipium flavium Egara. ${ }^{64}$

Respecto a Caldes, de nuevo, esta situación resulta más compleja de demostrar, pero aun así disponemos de otro epígrafe, del que también hemos hablado ya, donde se hace referencia a un cursus honorum municipal. ${ }^{65} \mathrm{~A}$ la vez, no sería un caso extraño en el noreste peninsular y, por ejemplo, tenemos otro conjunto termal, el de Caldes de Malavella (La Selva, Girona), identificado epigráficamente como Aquae Calidae y del que sabemos que fue promocionado a municipio en época flavia. Es éste un caso muy interesante y que puede aportar indicios que den soporte a nuestra interpretación sobre la evolución histórica de Caldes de Montbui. Así, estamos ante un núcleo del que la epigrafía, más escasa pero determinante, ha permitido documentar el nombre, Aquae Calidae, a partir de un epígrafe recuperado en la zona termal del Turó de Sant Grau, donde se enumera el cursus honorum de un Aquicaldensis. ${ }^{66} \mathrm{~A}$ la vez, se trata de un núcleo que aparece citado en las fuentes literarias y, de hecho, Plinio hace referencia a él describiéndolo como civitas stipendiaria.$^{67}$ Por otro lado, la presencia en el epígrafe citado de un cursus honorum municipal, junto a la perte-

62 IRC V, 235. Se trata de un miliario que marca la milla CX de la vía Augusta, realizado en el quinto consulado del emperador Tiberio. Sin duda, estaba en relación con el trazado de la vía que atravesaba precisamente la Layetania interior de forma longitudinal. Véase Oller Guzmán 2015, 234.

63 Véase supra, n. 9.

64 A través de dos epígrafes (IRC I, 66 y 69), en los que se registra un cursus honorum municipal y una dedicatoria a Antonino Pío donde se explicita el nombre del municipio.

65 IRC I, 42.

${ }_{6}$ IRC III, 9.

67 Plin. $H N 3.23$. 
nencia exclusiva de los personajes documentados epigráficamente a la tribu Quirina (también en el epígrafe IRC III, 8), ha permitido situar este núcleo entre los municipios romanos de promoción flavia. ${ }^{68}$ Creemos interesante remarcar el hecho de que, mientras que en Caldes de Malavella encontramos personajes locales exclusivamente adscritos a la tribu Quirina, en Caldes de Montbui esto sucede con la tribu Galeria. A pesar de no ser un elemento concluyente, generalmente se vinculan las promociones augusteas con la tribu Galeria y las flavias con la Quirina ${ }^{69}$ Ello podría reforzar la hipótesis de una promoción para Caldes de Montbui en época de Augusto, entrando sus ciudadanos en la tribu Galeria, una condición que ya no cambiaría al convertirse en municipio latino bajo los flavios. Ello también apoyaría la idea de una vinculación mayor con el primer emperador romano y sus herederos, dando más coherencia al patrocinio de Tiberio. En cambio, en el caso de Caldes de Malavella, la referencia de Plinio indicaría que, durante la primera mitad de siglo I d.C., estaríamos ante un núcleo estipendiario que sólo sería promocionado bajo la dinastía flavia, pasando sus ciudadanos por lógica a engrosar la tribu Quirina. ${ }^{70}$

Por tanto, y siendo conscientes de la parquedad de los datos, creemos plausible plantear la hipótesis de una promoción del núcleo de Caldes de Montbui como civitas termal en época de Augusto, con el objetivo de vertebrar una zona fundamental en el ámbito económico en el noreste peninsular como era el área vitivinícola de Lauro, en la parte central y oriental de la Layetania interior, paralelamente a un proceso idéntico sucedido en la parte occidental, con Egara como centro básico. ${ }^{71} \mathrm{La}$ evolución política de la zona y, especialmente, del Imperio, hizo que en época flavia ambos centros fuesen promocionados a municipios latinos, confirmando definitivamente su romanidad jurídica y consolidándose como centros territoriales de segundo orden, jerárquicamente sometidos a la colonia de Barcino. Dentro de este contexto, y si asumimos la procedencia de Caldes del epígrafe analizado, vemos que encaja mucho mejor el patrocinio a Tiberio. Un patrocinio que podría responder bien a una herencia por el papel de Augusto como promotor del núcleo, bien a la actividad del propio Tiberio en la zona, documentada en el ámbito viario. Como hipótesis personal, pensamos que quizá el establecimiento de esta relación de patrocinio en época de Tiberio cuadraría mejor con la lógica territorial y del propio epígrafe. Así, ya he-

68 Para un análisis detallado de las diferentes evidencias que permiten llegar a esta conclusión, véase Andreu Pintado 2004, 146.

69 Sobre esta cuestión y sus problemáticas, véase Castillo 1988, 233-243, y Andreu Pintado 2004, 128 y ss. Cf. Stylow 1995, 105-123; Fasolini 2012, 7-14.

70 Para más detalles acerca del caso de Caldes de Malavella, véase Merino i Serra et alii 1994; Llinàs i Pol et alii 2004, 69-89; Llinàs i Pol - Nolla Brufau 2011, 103-114.

71 En este sentido, creemos que la relación con la producción de vino de Lauro sería un aspecto fundamental para comprender cómo un núcleo relativamente pequeño como el de Caldes pudo llegar a acoger un honor de este tipo. Así, la importancia de esta producción vinícola-remarcada tanto por la referencia a su calidad por parte de Plinio como por la identificación de ánforas vinarias lauronenses en zonas del imperio como Roma, Ostia o Cartago- hace pensar en unas élites asociadas al control de dicha producción que, sin duda, ostentarían un nivel económico y social destacable. Y la prueba de ello la aportarían evidencias como la epigrafía anfórica, a través de la identificación de marcas en contenedores anfóricos hallados en los centros productivos de la misma Caldes, marcas en las que se documentan gentes como la Licinia (marcas SLL y LLQ, asociadas a un Lucius Licinius en los centros productivos de Sant Miquel de Martres y Can Cabot). Dicha gens sería una de las más poderosas de la zona layetana y estaría bien identificada entre las élites de núcleos como Barcino. Por ello pensamos que su identificación in situ en Caldes, en relación con una producción de vino de alta calidad, es significativa para poder dar credibilidad a este patrocinio de Tiberio. Para toda esta cuestión de la producción de vino en el área de Lauro, con abundante bibliografía sobre las marcas anfóricas, véase Oller Guzmán 2015, 248 y ss. 
mos visto que tanto la titulación del emperador como la referencia a Augusto divinizado confirman que el epígrafe fue elaborado a lo largo del gobierno de Tiberio; un período que precisamente la arqueología ha confirmado como de gran crecimiento económico en la zona de Caldes, en relación con la producción y exportación de vino. Por tanto, si a estos hechos añadimos las evidencias de actuaciones tiberianas en el territorio tarraconense (y en la misma Layetania, como mínimo a escala viaria), podemos pensar en un contexto en el cual el emperador Tiberio acabó de impulsar o consolidar iniciativas originarias del período augusteo en los territorios provinciales como Hispania. Y es en este contexto en el que creemos que tendría lógica pensar en el establecimiento de este vínculo entre el asentamiento romano de Caldes de Montbui y la familia imperial, impulsado probablemente por alguna de las poderosas gentes que actuaban en la zona y que controlaban su evolución económica y social, como los Licinii. $^{72}$

Obviamente, se trata de una hipótesis que no se puede confirmar con certeza, pero en cualquier caso, dicha posibilidad serviría para demostrar la importancia de este centro termal en el contexto regional, corroborada tanto por la intensa actividad económica desarrollada en su territorio circundante (vinculada a la producción de vino), ${ }^{73}$ como por su papel como centro salutífero de primer orden. ${ }^{74}$ Finalmente, dicho epígrafe también implicaría la existencia de algún tipo de edificio público de una cierta monumentalidad en donde habría sido expuesto ${ }^{75}$ Ello serviría para reforzar la hipótesis, basada en el registro arqueológico, de que estaríamos ante una civitas sine urbe: un núcleo sin una ciudad urbanísticamente definida, pero con la presencia de algunos edificios monumentales situados alrededor del conjunto termal, los cuales acumularían las funciones administrativas, jurídicas, económicas, religiosas, etc.

\section{Conclusiones}

Para acabar con este artículo, queremos exponer brevemente las principales conclusiones a las que hemos podido llegar tras analizar el epígrafe documentado en Caldes de Montbui.

72 Sin poder descartar, ni mucho menos, la opción de un patrocinio originalmente atribuido Augusto, teniendo en cuenta la importancia de la intervención de este emperador en el territorio layetano interior y su probable papel como impulsor de la promoción del núcleo romano de Caldes de Montbui como eje vertebrador de esta zona.

73 En este aspecto debe destacarse que, de nuevo, el análisis de las marcas anfóricas resulta clave al mostrar cómo, precisamente, el período tiberiano constituyó la época dorada de la producción de vino en la zona layetana, siendo una evidencia más del auge en la importancia de esta área en el ámbito económico y de la necesidad de vertebrarla y controlarla de forma intensa. Véase Berni Millet - Carreras Monfort 2001, 103-129.

74 Lo demostrarían los diversos epígrafes recuperados en el conjunto termal con dedicaciones de agradecimiento por la curación a divinidades como Apolo por parte de familias pudientes que provenían de algunas de las principales ciudades del noreste como Barcino, Baetulo o Tarraco. Sobre estos epígrafes Mayer - Rodà 1984, 2526; IRC I, 33-39.

75 Es en este sentido en el que se dirigen las apreciaciones de Cardon, quien, siguiendo a Fabre, Mayer y Rodà, identifica el epígrafe como parte de un friso de edificio público que considera debió estar erigido, sin duda, en el foro de la ciudad (Cardon 2004, 53). Evidentemente la autora desconoce la realidad del núcleo romano de Caldes, en el cual probablemente no existió un foro como tal, sino que más probablemente el área central del asentamiento romano se situaría en el espacio donde se encontraba el conjunto termal (actual Plaça de la Font del Lleó). El epígrafe, si continuamos con esta hipótesis, podría haber formado parte de alguna estructura de las mismas termas o de algún otro edificio público adyacente con funciones que podrían haber sido diversas. 
El conjunto de argumentos hasta aquí desarrollados nos lleva a concluir, aunque con cautela, que el epígrafe de Caldes podría documentar efectivamente un patrocinio cívico del emperador Tiberio en relación con esta zona del noreste de la Península Ibérica. Obviamente, existen elementos que dificultan dicha atribución, como la problemática acerca de su procedencia. Ahora bien, el hallazgo del epígrafe en un establecimiento religioso situado en las inmediaciones del centro termal, la contrastada importancia de este centro, su inserción en una zona de reputado prestigio económico a través de la producción del vino de Lauro y la existencia de un importante corpus epigráfico asociado, hacen que creamos perfectamente plausible una adscripción originaria del epígrafe a este asentamiento.

A la vez, la evolución política del núcleo romano de Caldes resulta fundamental para poder comprender este posible patrocinio. Los diferentes indicios y evidencias han hecho plantear la hipótesis de que estamos ante un núcleo promovido en época augustea, con el objetivo de vertebrar el territorio central y oriental de la Layetania interior, el cual habría sido ascendido al rango municipal en época flavia. Un caso paralelo y bien documentado sería Egara, en la zona layetana occidental. En este contexto, tendría sentido la existencia de un patrocinio por parte del emperador Tiberio en la civitas termal de Caldes, ya fuese como heredero del promocionador del núcleo, Augusto, o bien en base a su actuación en la zona, documentada en el ámbito viario.

Por tanto, en resumen, estamos ante un documento epigráfico de primer orden cuya importancia reside en el hecho de que, por un lado, se trataría de uno de los escasos patrocinia de Tiberio documentados en Hispania, y, por otro lado, porque aportaría datos fundamentales en relación a la evolución de un núcleo romano poco conocido hasta el momento como es el de Caldes de Montbui, constituido como magnífico ejemplo del proceso de romanización y de adaptación de las estructuras indígenas al Imperio Romano.

\section{Referencias bibliográficas}

Abascal, J. M. - Espinosa, U. (1989): La ciudad hispano-romana. Privilegio y poder, Logroño.

Aguilar, C. (2005): "Denominació d'origen Lauronensis. El vi del Vallès en època romana", Lauro 29, 5-12.

Alba Calzado, M. Á. - Mateos Cruz, P. (2006): “Augusta Emerita en época romana y tardoantigua (ss. I - VII)”, [en] Sánchez Montes - Rascón Marqués (eds.), 2006, 153-159.

Alföldy, G. (1965): “La politique provinciale de Tibère”, Latomus 24, 824-844.

Alvar, J. (1993): "Los cultos mistéricos de la Tarraconense", [en] M. Mayer (ed.), Religio deorum. Actas del Coloquio Internacional Epigrafia y sociedad en Occidente, Sabadell, 27-46.

Andreu Pintado, J. (2004): Edictum, municipium y lex: Hispania en época flavia (69-96 d.C.), (=BAR International Series 1293), Oxford.

Arrayás, I. (2006): "La instauración del modelo imperial en Hispania. La obra de César y Augusto" [en] T. Naco - I. Arrayás (eds.), War and territory in the Roman World (=BAR International Series 1530), Oxford, 179-201.

Arrayás, I. - Cortadella, J. - Ñaco, T. - Olesti, O. - Prieto, A. (2001): “Civitas y urbs en el nordeste hispánico: algunas reflexiones", [en] L. Hernández Guerra - L. Sagredo San 
Eustaquio - J. M. Solana Sáinz (eds), La Península Ibérica hace 2000 años. Actas del I Congreso Internacional de Historia Antigua, Valladolid, 311-317.

Barroso, C. - Esteve, J. A. - Martín, B. - Sánchez, P. D. (2003): "El conocimiento del territorio emeritense: la ocupación romana", Bolskan 20, 93-104.

Berni Millet, P. - Carreras Monfort, C. (2001): "El circuit comercial de Barcino: reflexions al voltant de les marques amfòriques", Faventia 23/1, 103-129.

Caballos Rufino, A.

(2004): "MAS REP 1990/85, otro fragmento de la Lex coloniae Genetiuae Iuliae", Zeitschrift für Papyrologie und Epigraphik 147, 211-216.

(2007): "La nueva tabla de la Lex coloniae Genitivae Iuliae. Avance Preliminar", [en] M. Mayer - G. Baratta - A. Guzmán (eds.), Provinciae Imperii Romani inscriptionibus descriptae. XII Congressus Internationalis Epigraphiae Graeca et Latinae (Barcelona, 3-8 Septembris 2002), Barcelona, 271-222.

Cardon, E. (2004): "Le patronat municipal en Bétique et en Tarraconaise sous les prémiers Julio-Claudiens. Un exemple de loyalisme dynastique", [en] J.-M. André (ed.), Hispanité et romanité (=Collection de la Casa de Velázquez 84), Madrid, 39-58.

Casabona Sebastián, J. F. - Pérez-Casas, J. A. (1994): "El foro de Caesaraugusta: un notable conjunto arquitectónico de época Julio-Claudia", [en] La ciutat en el món romà. Actes del XIVè Congrés Internacional d'Arqueologia Clàssica, Tarragona, vol. 2, 91-92.

Casas, A. - Cosentino, P. L. - Díaz, Y. - Fiandaca, G. - García, E. - Himi, M. - Lafuente, M. - Martorana, R. - Macias, J. M. - Menchón, J. J. - Muñoz, A. - Sala, R. - Teixell, I. (2008): “A la recerca del temple d'August a Tarragona: una experiència entre arqueologia i geofísica”, Cota Zero 23, 9-12.

Castillo, C. (1988): "La tribu Galeria en Hispania. Ciudades y ciudadanos", [en] J. González - J. Arce (ed.), Estudios sobre la Tabula Siarensis (=Anejos de AEspA IX), Madrid, 233-243.

Castillo, E. - Ruiz-Nicoli, B. (2008): “Iроnuba y su conjunto escultórico de época JulioClaudia", Romula 7, 149-186.

Cerrillo Martín de Cáceres, E. (2003): “La reorganización del territorio. Los paisajes de la romanización", [en] L. Abad Casal (ed.), De Iberia in Hispaniam. La adaptación de las sociedades ibéricas a los modelos romanos (=Lucentum. Anejos 10), Alicante, 37-52.

Díez de Velasco, F. (1996): "Invocaciones a Isis en ciudades de aguas (aquae) del occidente romano", [en] R. Rubio (ed.), Isis, nuevas perspectivas. Homenaje al Prof. Álvarez de Miranda (=Arys 4), Madrid, 143-153.

Duthoy, R. (1984-1986): "Le profil social des patrons municipaux en Italie sous le Haut Empire", Ancient Society 15-17, 121-154 (http://dx.doi.org/10.2143/AS.17.0.2011376).

Eilers, C. (2002): Roman patrons of Greek cities, Oxford (http://dx.doi.org/10.1093/acprof:o so/9780199248483.001.0001).

Escudero, F. de A. - Galve, M. P. (2006): "Vista de Caesaraugusta", [en] Sánchez Montes Rascón Marqués (eds.), 2006, 189-197.

Estrada, J. - Villaronga, L. (1967): "La Lauro monetal y el hallazgo de Cànoves”, Empúries 24, 134-194.

Fabre, G. - Mayer, M. - Rodà, I.

(1984): Inscriptions romaines de Catalogne. I. Barcelone (sauf Barcino), Paris (=IRC I). (1991): Inscriptions romaines de Catalogne. III. Gérone, Paris (=IRC III) (2002): Inscriptions romaines de Catalogne. Vol. V Supplèments aux volumes I-IV et instrumentum domesticum, Paris (=IRC V). 
Fasolini, D. (2012): Le tribù romane della Hispania Tarraconensis. L'ascrizione tribale dei cittadini romani nelle testimonianze epigrafiche, Milano.

Flórez, M. - Rodà, I. (2014): "Las vías romanas en Cataluña: el caso del Vallès Oriental (Barcelona)", [en] E. Boube - A. Bouet - F. Colleoni (eds.), De Rome à Lugdunum des Convenes. Hommages à Robert Sablayrolles (=Ausonius Éditions. Mémoires 35. Aquitania Suppl. 31), Bordeaux, 247-262.

Fortó Garcia, A. - Maese Fidalgo, X. (2012): "La Torre Roja: un jaciment ibèric i medieval (Caldes de Montbui, Vallès Oriental; Sentmenat, Vallès Occidental)", Tribuna d'Arqueologia 2009-2010, 113-152.

Fortó, A. - Maese, X. - Pelegero, B. - Pisa, J. - Vidal, A. (2005): "El poblat ibèric de la Torre Roja (Caldes de Montbui - Sentmenat)", Lauro 26-27, 5-18.

Garcia i Llinares, G. - Moro Garcia, A. - Tuset Bertrán, F. (2009): La seu episcopal d'Ègara. Arqueologia d'un conjunt cristià del segle IV al IX (=Institut Català d'Arqueologia Clàssica. Documenta 8), Tarragona.

Genera i Monells, M. (1994): "Dertosa: una ciutat romana", [en] La ciutat en el món romà. Actes del XIVè Congrés Internacional d'Arqueologia Clàssica, Tarragona, vol. 2, 171.

Genera i Monells, M. - Vianney Arbeloa, J. M. (1987): “L'estat actual de la investigació arqueològica sobre la Dertosa romana i la seva àrea d'influència", Tribuna d'Arqueologia 1986-1987, 81-90.

Gorges, J.-G. - Rodríguez Martín, F. G. (2005): "Los territorios antiguos de Mérida. Un estudio del territorium emeritense y de sus áreas de influencia", [en] T. Nogales Basarrate (ed.), Augusta Emerita. Territorios, espacios, imágenes y gentes en Lusitania romana (=MNAR. Monografías Emeritenses 8), Mérida, 93-128.

Guàrdia i Llorens, $\mathrm{M}$.

(2014): "Repensant Lauro: el projecte de recerca al poblat ibèric del Puig del Castell de Samalús (Cànoves i Samalús)", Ponències. Revista del Centre d'Estudis de Granollers $18,75-91$.

(2015): “A les portes de Lauro: el poblat ibèric de Puig del Castell de Samalús (Cànoves i Samalús)", Ponències. Revista del Centre d'Estudis de Granollers 19, 51-86.

Guerrero Arroyo, J. (1993): La Península Ibérica en época julio-claudia: las provincias Tarraconense y Lusitania, Tesis doctoral inédita, Universidad de Valladolid.

Harmand, L. (1957): Le patronat sur les collectivités publiques des origines au Bas-Empire. Un aspect social et politique du monde romain, Paris.

Henderson, M. I. (1942): “Julius Caesar and Latium in Spain”, Journal of Roman Studies 32, 1-13 (http://dx.doi.org/10.2307/296455).

Houdoy, J. (1876): Histoire du droit municipal, vol. I: De la condition des villes chez les romains, Paris.

Lintott, A. (1993): Imperium Romanum. Politics and Administration, London-New York.

Llinàs i Pol, J. - Merino i Serra, J. - Montalbán Martínez, C. (2004): "Les termes romanes de Sant Grau (Caldes de Malavella). Novetats arran de les excavacions de 2002", Quaderns de la Selva 16, 69-89.

Llinàs i Pol, J. - Nolla Brufau, J. M. (2011): “Aigua sagrada. El balneari del Puig de Sant Grau a Aquae Calidae (Caldes de Malavella, la Selva)", [en] A. Costa - L. Palahí - D. Vivó (eds.), Aquae Sacrae. Agua y sacralidad en la Antigüedad, Girona, 103-114.

Llorens Forcada, M. M. - Ripollès Alegre, P. P. (1998): Les encunyacions ibèriques de Lauro (=Estudis de Granollers i del Vallès Oriental 7), Granollers.

Lostal Pros, J. (1992): Los miliarios de la provincia Tarraconense (conventos tarraconense, cesaraugustano, cluniense y cartaginense), (=Institución Fernando el Católico 1314), Zaragoza. 
Macias, J. M. - Menchón, J. J. - Muñoz, A. - Teixell, I.

(2007a): "Excavaciones en la catedral de Tarragona y su entorno: avances y retrocesos de la investigación sobre el culto imperial", [en] T. Nogales - J. González (eds.), Culto imperial: política y poder (=L'Erma di Bretschneider. Hispania Antigua. Serie Arqueológica 1), Roma, 765-787.

(2007b): "L'arqueologia de la catedral de Tarragona. La memòria de les pedres", [en] J. Figuerola (ed.), La catedral de Tarragona. In sede, 10 anys del Pla Director de Restauració, Tarragona, 151-213.

Macias Solé, J. M. - Muñoz Melgar, A. - Peña Jurado, A. - Ramon Mas, M. - Teixell Navarro, I. (2012a): Praesidium, templum et ecclesia. Les intervencions arqueologiques a la Catedral de Tarragona 2010-2011. Memòria d'una exposició temporal, Tarragona.

Macias Solé, J. M. - Muñoz Melgar, A. - Teixell Navarro, I. (2012b): “Arqueologia a la nau central de la Catedral de Tarragona”, Tribuna d'Arqueologia 2010-2011, 151-173.

Malaise, M. (1984): "La diffusion des cultes égyptiens dans les provinces européennes de l'empire romain", $A N R W$ 17/3, 1615-1691.

Mangas, J. - Martínez Caballero, S. (2004): "Nuevas inscripciones romanas de Termes (Tiermes, Soria)", Veleia 21, 289-300.

Martín-Bueno, M. - Sáenz Preciado, J. C. (2004): “Los programas arquitectónicos de época julio-claudia de Bilbilis", [en] Ramallo Asensio (ed.), 2004, 257-276.

Mayer i Olivé, $\mathrm{M}$.

(2009): “Dertosa: colònia de Pertinax?”, Faventia 31/1-2, 61-69.

(2010): "El problema de las Aquae Calidae del norte del Conventus Tarraconensis", [en] F. Beltrán Lloris - J. L. García Alonso - C. Jordán Colera - E. R. Luján Martínez - J. Velaza Frías (eds.), Serta Palaeohispanica in honorem Javier De Hoz (=Palaeohispanica 10), 303-317.

Mayer, M. - Rodà, I.

(1984): La romanització del Vallès segons l'epigrafia, Sabadell.

(1987): "Epigrafia", Fonaments 6, 193-218.

Melchor Gil, E. (2014): "El patronato sobre comunidades cívicas hispanas en época augústea", Studia Historica, Historia Antigua 32, 249-279.

Merino i Serra, J. - Nolla i Brufau, J. M. - Santos Retolaza, M. (1994): Aquae Calidae. Presència romana a la Selva, Santa Coloma de Farners.

Miró i Alaix, C.

(1992a): "La arquitectura termal medicinal de época romana en Catalunya. Las termas de Caldes de Montbui como ejemplo", Espacio, tiempo y forma. Serie II. Historia Antigua 5, 255-276 (https://doi.org/10.5944/etfii.5.1992.4194).

(1992b): "Les termes romanes de Caldes de Montbui", Arraona 10, 11-29.

Nicols, J.

(1978): "The emperor and the selection of the patronus civitatis. Two examples", Chiron 8, 429-433.

(2014): Civic patronage in Roman Empire (=Mnemosyne, Suppl. 365), Leiden.

Olesti Vila, O. - Garcia Llinares, G. (1999): “Terrassa i el seu territori a l'edat antiga: estat de la qüestió i noves perspectives de recerca", Terme 14, 13-23.

Oller Guzmán, J.

(2009): "El municipi romà d'Ėgara: antecedents, constitució i evolució", Terme 24, 189-208. (2014): "La civitas sine urbe y su función de vertebración en el territorio provincial hispano: los casos de Egara y Caldes de Montbui", Pyrenae 45/1, 89-110 (https://doi. org/10.1344/Pyrenae2014.vol45num1.4). 
(2015): El territorio y poblamiento de la Layetania Interior en época antigua (ss. IV a.C.I d.C.), (=Universitat de Barcelona. Col·lecció Instrumenta 51), Barcelona.

(2016): "La Layetania interior en época augústea: un ejemplo de reordenación territorial en la Hispania Citerior Tarraconense", Dialogues d'Histoire Ancienne 42/1, 137-175.

Pascual, R. (1998): "La Lauro vinícola", [en] M. Mayer - J. M. Nolla - J. Pardo (eds.), De les estructures indígenes a l'organització provincial romana de la Hispània Citerior. Homenatge a Josep Estrada i Garriga (=Ítaca. Annexos 1), Barcelona, 149-162.

Prieto Arciniega, A.

(1997): “La relación entre Égara y Barcino en época romana y visigòtica”, [en] M. I. Loring García (ed.), Historia social, pensamiento historiográfico y Edad Media. Homenaje al profesor Abilio Barbero de Aguilera, Madrid, 209-215.

(2008): "La organización territorial del nordeste de la Hispania Citerior", [en] J. Uroz - J. M. Noguera - F. Coarelli (eds.), Iberia e Italia: modelos romanos de integración territorial, Murcia, 25-42.

Ramallo Asensio, S. F. (ed.), (2004): La decoración arquitectónica en las ciudades romanas de occidente (Actas del Congreso Internacional celebrado en Cartagena entre los días 8 y 10 de octubre de 2013), Murcia.

Rodríguez Oliva, P. - Beltrán Fortes, J. (1986): "Una inscripción dedicada a Tiberio en Carissa Avrelia", Baetica 9, 219-225 (http://dx.doi.org/10.24310/BAETICA.1986.v0i9.819).

Roig i Buxó, J.

(2002): "Indicis arqueològics del pas de la via Augusta per Sabadell (Vallès Occidental): la intervenció arqueològica a la riba esquerra del riu Ripoll", Arraona 26, 76-91.

(2004): "La intervenció arqueològica a la riba esquerra del riu Ripoll (Sabadell, Vallès Occidental)", [en] Jornades d'Arqueologia i Paleontologia 2001, Barcelona, 809-823.

Salinas de Frías, M. (1995): El gobierno de las provincias hispanas durante la República romana, 218 - 27 a.C. (=Universidad de Salamanca. Acta Salmanticensia. Estudios Históricos y Geográficos 96), Salamanca.

Sánchez Montes, A. L. - Rascón Marqués, S. (eds.), (2006): Civilización. Un viaje a las ciudades de la España Antigua, Alcalá de Henares.

Santos Yanguas, J. (1989): “Colonización y municipalización de Hispania desde Tiberio a los flavios”, [en] Aspectos de la colonización y municipalización de Hispania (=Cuadernos Emeritenses 1), Mérida, 107-132.

Solana Sáinz, J. M. - Sagredo San Eustaquio, L.

(2006): La red viaria romana en Hispania: siglos I-IV d.C. (=Universidad de Valladolid. Serie Historia y Sociedad 119), Valladolid.

(2008): La política viaria en Hispania: siglos I-II d.C. (=Universidad de Valladolid. Serie Historia y Sociedad 130), Valladolid.

Stylow, A. (1995): “Apuntes sobre las tribus romanas en Hispania”, Veleia 12, 105-123.

Trillmich, W. (2004): "Los programas arquitectónicos de época julio-claudia en la colonia Augusta Emerita”, [en] Ramallo Asensio (ed.), 2004, 321-336. 